\title{
Development of Stem-Loop Real-Time PCR Technique for miRNA-141 Expression Analysis in Nasopharyngeal Carcinoma
}

\author{
Thuan Duc Lao and Thuy Ai Huyen Le* \\ Faculty of Biotechnology, Department of Pharmaceutical and Medical Biotechnology, Ho Chi \\ Minh City Open University, Ward Co Giang, District 1, HCMC, Ho Chi Minh City, Vietnam; \\ ducthuanlao87@gmail.com, thuan.ld@ou.edu.vn, thuy.lha@ou.edu.vn
}

\begin{abstract}
Background: The deregulation of miRNA-141 (miR-141) has been reported to be involved in development and progression of many various types of human cancers. Previously, it has been demonstrated that miR-141 served as the oncogenic role in nasopharyngeal carcinoma in different countries. However, in Vietnam, to our knowledge, there are limit study of the types of evaluation of miR-141 expression. Objective: For the aims to find out the novel diagnosis strategies and promising therapies for many human based on the expression of miRNA, we the stem-loop qRT-PCR method described here is to detect mature miRNA-141 in a fast, specific, accurate, and reliable manner. Methods: StL-miR-141, known as stem-loop RT primer is applied for Reversed transcriptase assay, followed by Real-time PCR assay using StL-F, STL-R primer and SYBRGreen. Results: Experimentally, this method was applied in detection of miR-141 in Vietnamese NPC biopsy samples, as the results, 5 of six NPC were positive to miR-141 expression. Moreover, the electrophoresis analysis was performed to evaluate the Specificity. The assay has shown high specific for miR-141 detection. Conclusion: We successfully designed the stem-loop RT primer, StL-F, STL-R primer, might be used as a rapid, simple method for the detectionwith a wider scope to apply this method onto the Vietnamese population.
\end{abstract}

Keywords: miRNAs, miR-141, Stem-Loop RT PCR

\section{Introduction}

Vietnam is country with the high nasopharyngeal carcinoma incidence and mortality rate within 86,691 cases (Age-standardized rate - ASR $=1.2 / 100,000$ ) and $50,831$ (ASR $=0.7 / 100,000)$ deaths $^{1,2}$. Although many improvements have been increased around for recent decades, most patients still reach an advanced stage, owing to the ambiguous symptoms $s^{3-5}$. Therefore, improved identification of potential biomarkers associated with NPC progression is essential for the development of new diagnosis strategies and promising therapies.
microRNAs (miRs, miRNAs), typically represent $\sim 20$ nucleotides in length, which can bind to sequences in 3'-untranslated region (3'-UTR) of their target mRNAs, resulting block their translation, are the abundant class of evolutionarily conserved, small non-coding $\mathrm{RNAs}^{6-8}$. Resulting to the affections on numerous cellular processes including cell proliferation, differentiation apoptosis and metabolism ${ }^{8}$. Recent years, growing evidences indicated the regulation of miRNAs have been reported as both oncogenes and tumor suppressor genes that the abnormal expression of miRNAs contributes to many human tumor pathogenesis, including Naso-Pharyngeal Carcinoma

*Author for correspondence 
(NPC) and other cancers ${ }^{9-16}$. Many reports demonstrated that the present of miR-141 may be a potential target for the therapy of NPC.

miRNA-141 (miR-141), known as the oncogenic microRNA, locates at 12q13.31 (has-miR-141: Accession number: MI0000457, nt: 6964097-6964191, [+]). The dysregulation of miR-141 has been reported in many human types of cancers, such as Hepatocellular carcinoma, renal cell carcinoma, breast cancer, ovarian cancer, as well as NPC ${ }^{5,16-18}$.

Notably, miRNAs are the small noncoding RNAs ( 20 nucleotide in length) and lack of a common feature like a poly(A) tail in $3^{\prime}$ region, present in eukaryotic cells, therefore, it is necessary to develop the method for detecting mature miRNA in fast, specific, accurate and reliable manner. Up to date, many developed RT-qPCR methods of miRNA detection, including poly(A)tailing RT-PCR, primer-extension RT-qPCR, stem-loop qPCR, miQPCR, etc. have become established. To our knowledge, no studies of the types of miR-141 expression evaluation in Vietnamese NPC patients have been reported to date. Moreover, there has been no low-cost and simple methods for detection of miRNAs. It meant that it is high cost for consumed detection miRNAs kit applied in Vietnam. In the light of this, the aim of the study was to design a simple stem-loop qPCR method to detect miR-141 in Vietnamese NPC samples with a wider scope to apply this method onto the population.

\section{Materials and Methods}

\subsection{Designing Primers, Stem-Loop Primer}

miR-141 sequence was collected from miRNA database (www.mirbase.org) by accession number: MI0000457. The other miRNA-141 family members, including miR-200a, miR-200b, miR-200c and miR-429 were also collected within following accession number: MI0000737, MI0000342, MI0000650, and MI0001641. Then, the alignment was done by BioEdit Sequence Alignment Editor Software. The primers includes: stem-loop primer (StL-miR-141) for the first stage, forward and reversed primer (StL-F, StL-R) for the second stage, were designed to amplify only miR-141. Secondary structure of stem- loop primer was analysed by mfold analysis tool (http:// unafold.rna.albany.edu/?q=mfold/). The characteristic of primers were evaluated by using IDT oligo analyzer.

\subsection{Sample Collection}

Institutional Ethics Board approval was obtained from the Medical Ethics Committee of the Cho Ray Hospital, Ho Chi Minh City, Vietnam (The decision number of the permission from Ethical committee: 516/BVCR-HDDD, Cho Ray Hospital, Ho Chi Minh City, Vietnam). All the samples used in this study were agreed by Cho Ray Hospital and obtained from all participants in current study. The patients are required to be agreed and sign on the consent forms.

For current initial study, six NPC clinical biopsy tissues were archived and admitted from the Cho Ray Hospital, Vietnam. All of those samples were submitted to the histopathological department, subsequently, were proved histologically to have NPC by Immunohistochemistry (IHC) confirmed.

\subsection{Total RNA Isolation, Reversed Transcriptase and miR-141 Detection by using Stem-Loop Real-Time PCR}

miRNAs were isolated from NPC biopsy samples and non-cancerous samples by applying mirVanaTM miRNA Isolation Kit, with Phenol (Ambion, Life Technology) according to the manufacturer's instructions.cDNA was reverse transcribed from approximately $1 \mu \mathrm{g}$ of Total RNA by using Stem-loop primers. Then, $100 \mathrm{ng}$ RT reaction products were used to detect of miR-141 by qualitative Real-time PCR (qRT PCR) assay with StL-F and StL-R primers. Specificity was confirmed by gel electrophoresis analysis.

The stem-loop Reversed Transcriptase PCR was performed in $20 \mu$ mixture volume by using RevertAid First Strand cDNA Synthesis Kit (Thermo Scientific, K1622) with the random primer was replaced by stemloop primer. For the second stage, the Realtime-PCR was done in $20 \mu \mathrm{l}$ mixture volumes by using $2 \mathrm{X}$ SYBR Green qPCR Master Mix (Thermo Scientific, K0221) and StL-F, StL-R primer. The thermal cycle for RT assay and Realtime assay were shown in Table 1. 
Table 1. Thermal assay for RT and Real-time reaction

\begin{tabular}{|c|c|c|}
\hline & Temperature $\left({ }^{\circ} \mathbf{C}\right)$ & Time \\
\hline \multicolumn{3}{|c|}{ Reversed transcriptase assay } \\
\hline Step 1 & 25 & $5 \mathrm{mins}$ \\
\hline Step 2 & 42 & $60 \mathrm{mins}$ \\
\hline Step 3 & 70 & $5 \mathrm{mins}$ \\
\hline \multicolumn{3}{|c|}{ Real-time assay } \\
\hline \multirow{2}{*}{ Step 1 } & 95 & $2 \mathrm{mins}$ \\
\hline \multirow{3}{*}{$\begin{array}{c}\text { Step 2 (40 } \\
\text { cycles) }\end{array}$} & 95 & $5 \mathrm{sec}$ \\
\cline { 2 - 3 } & 60 & $10 \mathrm{sec}$ \\
\cline { 2 - 3 } & 72 & $10 \mathrm{sec}$ \\
\hline
\end{tabular}

\section{Results}

\section{1 miR-141 Sequence, Designing the Primer (StL-F, StL-R) and Stem-Loop Primer (StL-miR-141)}

miR-141 sequence was collected from miRNA database. The length of miR-141 is 95 nucleotides, contains passenger strand (hsa-miR-141-5p, previously called
miR-141*) and miR-141 mature strand (hsa-miR-141-3p) (Figure 1A, B). The other member of family of miR-200 was also collected and aligned each other to indicate the homology in their seed regions. Notably, the nucleotides, located on the 3 ' region, of them were different, thus, it was used to design the Stem-loop specific primer to amplify the miR-141-3p (Figure 1C).

Stem-loop RT primer, named as StL-miR-141, was specific to target RT of miR-141. Specific forward primer and reversed primer, named as StL-F, StL-R, were designed for Real-time PCR (Table 2).

\subsection{Characteristics of StL-miR-141, StL-F and StL-R}

The secondary structure of StL-miR-141 was analyzed by mfold, shown in Figure 2A. According to the structure of StL-miR-141, it forms the hairpin structure within the non- complementary stem-loop region, and the noncomplementary 6 nucleotides at the 3 ' end. The sequence at the 3' end (5'-CCATCT-3') of the StL-miR-141 primer is a specifically complementary to mature miR-141 at the sequence ( $3^{\prime}$-GGUAGA-5'), and the elongated sequence from the specific $3^{\prime}$ end is underlined (Figure $2 \mathrm{~B}$ ). The

(A)

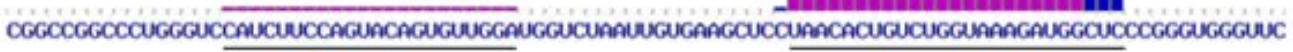

$$
\begin{aligned}
& \text { miR-141-5p } \\
& \text { miR-141-3p }
\end{aligned}
$$

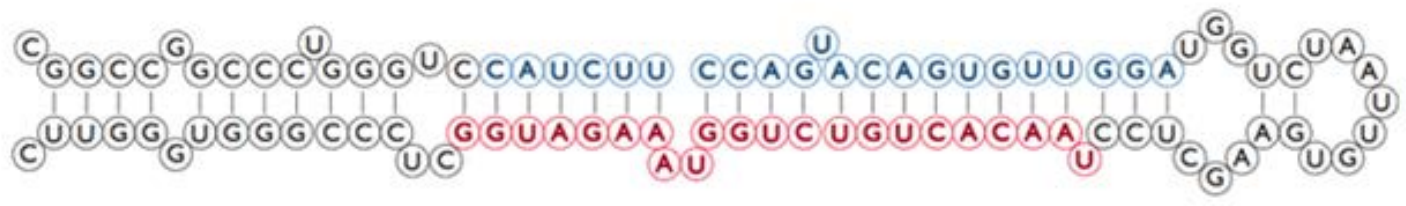

(B)
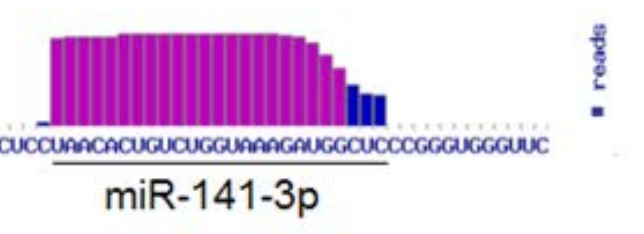

(B)

(C)



Figure 1. (A) The sequence of precursor miR-141 (Accession number: MI0000457), contains the mature miR-141$3 p$, and the passenger strand miR-141-5p; (B) Schematic representation of the secondary structure of the pre-miR-141 hairpin; (C) Sequence of the five members of miR-200 family. The seed sequence is underlined, and the two subfamilies are indicated by Sub-1 and Sub-2 bar. The different nucleotides, located 3 ' region, of five members were indicated in the square bracket. 
Table 2. Designed Stem-loop RT, specific forward primer and reversed primer

\begin{tabular}{|c|c|c|}
\hline \multicolumn{2}{|c|}{ Primer } & Sequence (5'-3') \\
\hline StL-miR-141 & $\begin{array}{c}\text { Stem-loop RT } \\
\text { primer }\end{array}$ & $\begin{array}{c}\text { TCTACCCAGCATAGGTC } \\
\text { ACGCTTATGGAGCCTGG } \\
\text { GACGTGACCTATGCTG }\end{array}$ \\
\hline StL-F & $\begin{array}{c}\text { Forward } \\
\text { primer }\end{array}$ & $\begin{array}{c}\text { GCGATTGTGACAGACC } \\
\text { ATTTC }\end{array}$ \\
\hline StL-R & $\begin{array}{c}\text { Reversed } \\
\text { primer }\end{array}$ & TTCAGCATAGGTCAC \\
GTCC
\end{tabular}

specificity of StL-miR-141 is to amplify the miR-141-3p sequence, and not to amplify other sequences of the miR200 family due to the differences were observed on the 3 ' end of miRNAs (Figure 1C). The StL-F and StL-R primer were designed for Real-time PCR and yield the product of 71 bps in length (Figure 2C). Moreover, nucleotide BLAST for the determination of the specificity of each primer showed that no complemented to any other sequences, except its pertaining target miR-141.

\subsection{Detection of miR-141 in Clinical NPC Samples and Non-Cancerous Samples by Stem-Loop Real-Time Assay}

The total RNAs of six NPC biopsy samples were extracted by mirVanaTM miRNA Isolation Kit (Ambion, Life Technology). cDNA was reversed transcriptase by using stem-loop reserved primer (StL-miR-141). Realtime PCR amplification was performed with Sybr ${ }^{\circledR}$ No-Rox mix, and StL-F, StL-R primer. As the results, shown in Figure $3 \mathrm{~A}$, we detected positive miR-141 expression in 5 of 6 of NPC biopsy samples (counting for 83.33\%). Specificity was confirmed by gel electrophoresis analysis (Figure 3B). The Realtime-PCR products obtained in the band of $72 \mathrm{bps}$ in length, which was according to the analysis of StL-F and StL-R primer, which shown in (Figure 2C).

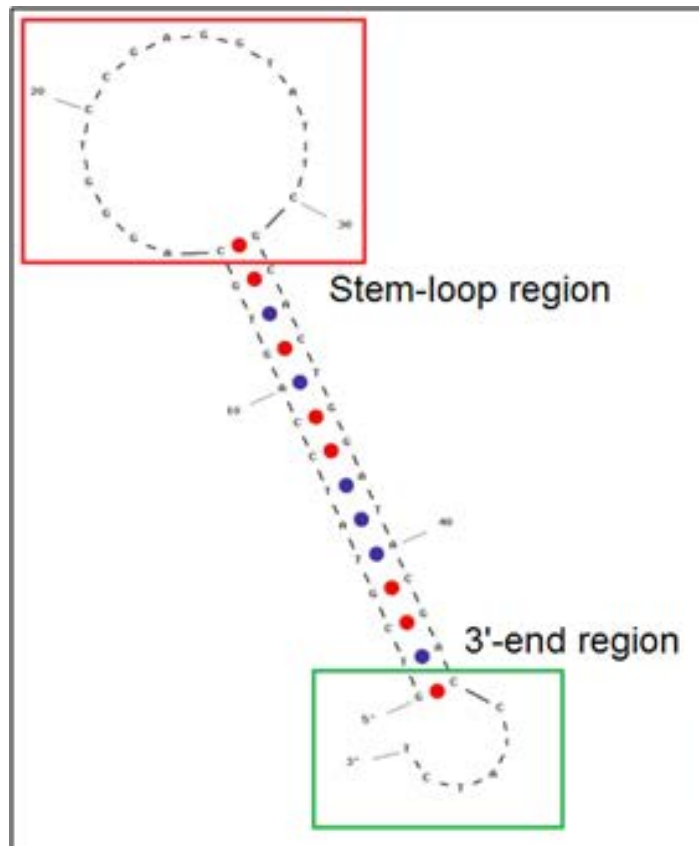

(A)

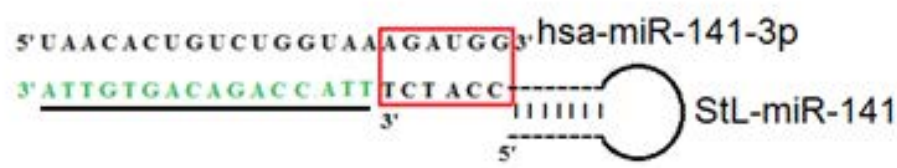

(B)

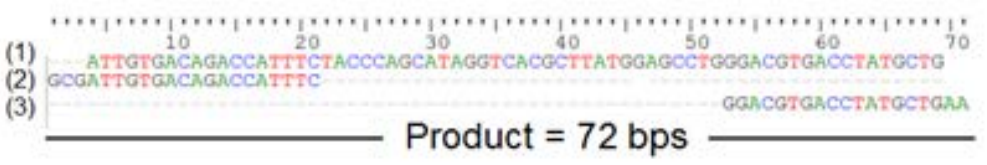

(C)

Figure 2. (A) The secondary of StL-miR-141; (B) Schematic representation of complementary sequence between StL-miR-141 and has-miR-141-3p. The elongated sequence from the specific 3' end is underlined; (C) The StL-F and StL-R amplified the 71-bps in length based on the template obtained from the stem-loop RT assay. (1) the StL-miR-141 product, (2) StL-F sequence, (3) StL-R sequence. 


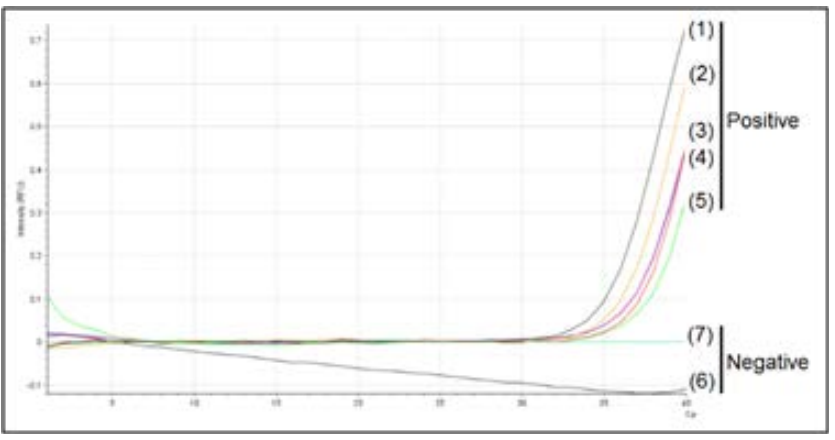

A

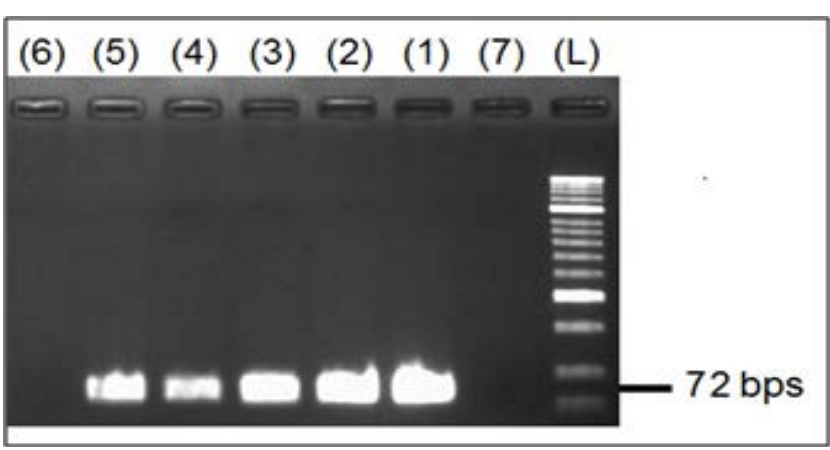

$\mathrm{B}$

Figure 3. (A) Amplification curve of miR-141 expression detection; (B) Specificity of miRNA real-time PCR product determined bygel electrophoresis. (1) à (6): NPC biopsy samples; (7) Negative control; (L) 50 bps ladder.

\section{Discussion}

Up to date, accumulating evidences indicated the dysregulation of miRNAs has crucial roles in the progression of various human cancers ${ }^{8,9,16,19}$. miRNAs signature has been proposed as the potential way of being prognostic or therapeutic target for human cancer. Additionally, as the number of miRNAs continues to increase, it is necessary to remain important tools for identification of differential expression of miRNAs. Several well-developed RT-qPCR methods of miRNA detection, such as poly(A)-tailing RT-PCR, primer-extension RT-qPCR, stem-loop RT-qPCR, miQPCR, each with its own advantages and disadvantage. Notably, in Vietnam, no study about of miRNAs detection in Vietnamese human cancer patients has been reported. Therefore, in current study, we investigated the stem-loop RT qPCR method to apply in detection of miRNAs expression in Vietnamese population. For this purpose, miR-141, which has been demonstrated as the oncogenic role in various human cancers, including NPC, was chosen to be the miRNA target for designing the stem-loop RT primers. In current study, stem-loop RT-qPCR detection was initiated by Reverse Transcription (RT) with 50-nucletode stemloop RT primer. The StL-miR-141 was designed to target specific reversed transcriptase of miR-141-3p based on the non-complementary 6 nucleotides (5'-CCATCT-3') at the $3^{\prime}$ end. After the RT assay, the size of cDNA sequence, which was amplified by the specific StL-miR-141 primer, was elongated to $67 \mathrm{bps}$. The last stage of stem-loop RT PCR was performed with the couple specific primer to the 67-bps cDNA obtained from previous step. Interestingly, using the StL-F, StL-R primer in the Real-time PCR amplification yielded the amplified product within 72-bps in length, and make this technique more feasible in which based on SYBR-Green. In order to test the capability of our presented method, it was initially applied in a small amount of NPC biopsy samples collected at local hospital. Experimentally, the stem-loop RT method analysis showed the positive of miR-141 expression was 5 of 6 cases, counting for $83.33 \%$. The Specificity of that product were confirmed by gel electrophoresis. The number of miR-141 expression positive case, which observed in gel agarose electrophoresis, were the similar to the results of Real-time PCR assays. Therefore, we concluded that our presented methods, based on the designed StL-miR-141, StL-F, StL-R, can detect the expression of miR-141 in the clinical samples. According to Chen et al. (2005), stemloop RT-qPCR is a highly efficient, sensitive and specific method of miRNA detection. It could be explained that neither polyadenylation nor adaptor ligation is required, thus, this method is considered as more convenient, less expensive than the other method $^{20}$. In general we successfully in developing the stem-loop RT qPCR method for detection of miR-141 expression based on this designed principles of this method, it will be easily to expand to develop each specific set primer for each interested miRNAs, and it could be further applied in the research relevant to miRNA detection. However, for the kit development based on those designed primers, it is required to have the assay, in which the sensitivity, limit of detection, comparison with other detection of miRNA expression, could be tested. 


\section{Conclusion}

In this study, we described the designed stem-loop RT PCR method for detection of miR-141 expression. Initially, this method was applied in detection of miR-141 in NPC biopsy samples, as the results, the stem-loop Realtime method analysis showed the positive of miR-141 expression was 5 of 6 cases. The Specificity of miRNA realtime PCR product was confirmed bygel electrophoresis. To our knowledge, this is the first publication of its type: designing the stem-loop RT method to simplify evaluates the expression miR-141 in Vietnamese NPC samples with a wider scope to apply this method onto the large samples.

\section{Acknowledgments}

We wish to express our thanks for the research project sponsored by the Ministry of Education and Training, Vietnam; Ho Chi Minh City Open University; We also thank all the recruited participants in this work and Dr. Nguyen Trong Minh, Dr. Nguyen Huu Dung, all the otorhinolaryngology staff members of in Cho Ray Hospital, Ho Chi Minh City, for collecting the samples used in these studies. We are thankful to the participants in our study and appreciated the contributions of the staffs from Molecular Biology Laboratory, Ho Chi Minh City Open University.

Declaration of interest: None

\section{Reference}

1. GLOBOCAN. Estimated cancer incidence, mortality and prevalence worldwide in 2012. 2012. http://globocan.iarc. fr/Pages/fact_sheets_population.aspx.

2. Mahdavifar N, Ghoncheh M, Mohammadian-Hafshejani A, Khosravi B, Salehiniya H. Epidemiology and inequality in the incidence and mortality of nasopharynx cancer in Asia. Osong Public Health Res. Perspect. 2016; 7(6):36072. https://doi.org/10.1016/j.phrp.2016.11.002. PMid: 28053841, PMCid: PMC5194228.

3. Epstein JB, Jones CK. Presenting signs and symptoms of nasopharyngeal carcinoma. Oral Surg. Oral. Med. Oral. Pathol. 1993; 75(1):32-36. https://doi.org/10.1016/00304220(93)90402-P.

4. Hao SP, Tsang NM, Chang KP, Ueng SH. Molecular diagnosis of nasopharyngeal carcinoma: Detecting LMP-1 and EBNA by nasopharyngeal swab. Otolaryngol Head Neck Surg. 2004; 131(5):651-54. https://doi.org/10.1016/j. otohns.2004.04.013. PMid: 15523443.
5. Liu Y, Zhao R, Wang H, Luo Y, Wang X, Niu W, et al. miR-141 is involved in BRD7-mediated cell proliferation and tumor formation through suppression of the PTEN/ AKT pathway in nasopharyngeal carcinoma. Cell Death Dis. 2016; 7:e2156. https://doi.org/10.1038/cddis.2016.64. PMid: 27010857, PMCid: PMC4823963.

6. Lee RC, Feinbaum RL, Ambros V. The C. elegansheterochronic gene lin-4 encodes small RNAs with antisense complementarity to lin-14. Cell. 1993; 75(5):843-54. https:// doi.org/10.1016/0092-8674(93)90529-Y.

7. Ha M, Kim VN. Regulation of microRNA biogenesis. Nat. Rev. Mol. Cell. Biol. 2014; 15(8):509-24. https://doi.org/10.1038/ nrm3838. PMid: 25027649.

8. Bartel DP. MicroRNAs: Genomics, biogenesis, mechanism, and function. Cell. 2004; 116(2):281-97. https://doi. org/10.1016/S0092-8674(04)00045-5.

9. Calin GA, Croce CM. MicroRNA signatures in human cancers. Nat. Rev. Cancer. 2006; 6(11):857-66. https://doi. org/10.1038/nrc1997. PMid: 17060945.

10. Faraoni I, Antonetti FR, Cardone J, Bonmassar E. miR-155 gene: A typical multifunctional microRNA. Biochim. Biophys. Acta. Biochim. Biophys. Acta. 2009; 1792(6):497-505. https:// doi.org/10.1016/j.bbadis.2009.02.013. PMid: 19268705.

11. Macfarlane LA, Murphy PR. Micro RNA: Biogenesis, function and rolein cancer. Curr. Genomics. 2010;11(7):53761. https://doi.org/10.2174/138920210793175895. PMid: 21532838, PMCid: PMC3048316.

12. Du ZM, Hu LF, Wang HY, Yan LX, Zeng YX, Shao JY, et al. Upregulation of MiR-155 in nasopharyngeal carcinoma is partly driven by LMP1 and LMP2A and down regulates a negative prognostic marker JMJD1A. PLoS One. 2011; 6:e19137. https://doi.org/10.1371/journal.pone.0019137. PMid: 21541331, PMCid: PMC3082546.

13. Lovat F, Valeri N, Croce CM. MicroRNAs in the pathogenesis of cancer. Semin. Oncol. 2011; 38(6):724-33. https://doi.org/10.1053/j.seminoncol.2011.08.006. PMid: 22082758.

14. Amin M, Lam AK. Current perspectives of mi-RNA in oesophagealadenocarcinoma:Rolesinpredictingcarcinogenesis, progression and values in clinical management. Exp. Mol. Pathol. 2015; 98(3):411-18. Doi: 10.1016/j.yexmp.2015.03.002. https://doi.org/10.1016/j.yexmp.2015.03.002. PMid: 25746664.

15. Lee KT, Tan JK, Lam AK, Gan SY. MicroRNAs serving as potential biomarkers and therapeutic targets in nasopharyngeal carcinoma: A critical review. Crit. Rev. Oncol. Hematol. 2016; 103:1-9. https://doi.org/10.1016/j.critrevonc.2016.04.006. PMid:27179594.

16. Lao DT, Truong KP, Le HAT.miRNA-141 as the Biomarker for Human Cancers. JPRHC. 2018; 10:42-49. https://doi. org/10.18311/ajprhc/2018/21486. 
17. Li G, Huang M, Cai Y, Ke Y, Yang Y, Sun X. miR 141 inhibits glioma vasculogenic mimicry by controlling EphA2 expression. Mol. Med. Rep. 2018(2); 18:1395-1404. https://doi.org/10.3892/ mmr.2018.9108.

18. Lin L, Liang $\mathrm{H}$, Wang $\mathrm{Y}$, Yin $\mathrm{X}, \mathrm{Hu} \mathrm{Y}$, Huang J, et al. microRNA-141 inhibits cell proliferation and invasion and promotes apoptosis by targeting hepatocyte nuclear factor-3 $\beta$ in hepatocellular carcinoma cells. BMC cancer. 2014; 14:879. https://doi.org/10.1186/1471-2407-14-879. PMid: 25425543 , PMCid: PMC4289273.
19. Croce CM, Calin GA. miRNAs, cancer, and stem cell division. Cell. 2005; 122:6-7. https://doi.org/10.1016/j. cell.2005.06.036. PMid: 16009126.

20. Chen C, Ridzon DA, Broomer AJ, Zhou Z, Lee DH, Nguyen JT, et al. Real-time quantification of microRNAs by stemloop RT-PCR. Nucleic Acids Res. 2005; 33(20):e179. https://doi.org/10.1093/nar/gni178. PMid: 16314309, PMCid: PMC1292995. 